\title{
Control of Multi-Resistant Bacteria and Ventilator-Associated Pneumonia: Is It Possible with Changes in Antibiotics?
}

\author{
Elisa M. Jukemura ${ }^{1}$, Marcelo N. Burattini ${ }^{1}$, Carlos A.P. Pereira ${ }^{1}$, Alfésio L.F. Braga ${ }^{2}$ and Eduardo A.S. Medeiros ${ }^{1}$ \\ ${ }^{1}$ Infectious Diseases Division, Internal Medicine Department, Federal University of São Paulo School of Medicine; ${ }^{2}$ Collective Health Pos- \\ graduation Program, Catholic University of Santos; São Paulo, SP, Brazil
}

\begin{abstract}
Potent antimicrobial agents have been developed as a response to the development of antibiotic-resistant bacteria, which especially affect patients with prolonged hospitalization in Intensive Care Units (ICU) and who had been previously treated with antimicrobials, especially third-generation cephalosporins.This study was to determine how changes in the empirical treatment of infections in ICU patients affect the incidence of Gram-negative bacteria species and their susceptibility to antimicrobials, and examine the impact of these changes on nosocomial infections. A prospective interventional study was performed in a university hospital during two periods: 1) First period (September 1999 to February 2000); and 2) Second period (August 2000 to December 2000); empirical treatment was changed from ceftriaxone and/or ceftazidime in the first period to piperacillin/tazobactam in the second. ICU epidemiological and infection control rates, as well as bacterial isolates from upper airways were analyzed. Ceftazidime consumption dropped from 34.83 to $0.85 \mathrm{DDD} / 1000$ patients per day ( $\mathrm{p}=\mathbf{0 . 0 0 4}$ ). Piperacillin/tazobactam was originally not available; its consumption reached $157.07 \mathrm{DDD} / 1000$ patients per day in the second period $(\mathrm{p}=\mathbf{0 . 0 0 0 2})$. Eighty-seven patients and 66 patients were evaluated for upper airway colonization in the first and second periods, respectively. There was a significant decrease in the incidence of $K$. pneumoniae $(\mathrm{p}=0.004)$ and $P$. mirabilis $(\mathrm{p}=0.036)$, restoration of $K$. pneumoniae susceptibility to cephalosporins $(\mathbf{p}<\mathbf{0 . 0 0 0 1})$ and reduction of ventilator-associated pneumonia rates $(\mathbf{p}<0.0001)$. However, there was an increase in $P$. aeruginosa incidence $(\mathrm{p}=0.005)$ and increases in ceftazidime $(\mathrm{p}=0.003)$ and meropenem $(\mathrm{p}<\mathbf{0 . 0 0 0 1})$ susceptibilities. Changing antimicrobial selective pressure on multi-resistant Gram-negative bacteria helps control ventilator-associated pneumonia and decreases antimicrobial resistance.

Key-Words: Ventilator associated pneumonia, multi-resistant bacteria, infection control.
\end{abstract}

Since the introduction of penicillin, bacteria have been shown to have the capacity of developing resistance when exposed to antibiotics. There is considerable evidence that previous exposure to antibiotics plays an important role in the development of resistance [1].

Nowadays, the problem of resistance is of great concern in the hospital setting due to multi-resistant $S$. aureus, Enterococcus spp., Acinetobacter spp., and Pseudomonas spp., giving birth to the so-called post-antibiotic era [2].

There is some evidence that increased use of thirdgeneration cephalosporins leads to resistance in Gramnegative bacilli, especially Citrobacter, Enterobacter, Serratia and Providencia species, and also to emergence of extendedspectrum beta-lactamase (ESBL)-producing bacteria $[3,4]$. Once this happens, the use of carbapenems, which can induce resistance in $P$. aeruginosa and Acinetobacter spp. [5], becomes mandatory.

Hospital São Paulo, a large university hospital in Brazil, experienced an increase in ESBL-producing Klebsiella pneumoniae in 1995, when $39 \%$ of the isolates were found to be resistant to cephalosporins. These bacteria were not clonal; their spread was thought to be due to the use of thirdgeneration cephalosporins [6]. This led to increased use of carbapenems, favoring an increase in the incidence of Received on 05 February 2007; revised 28 July 2007.

Address for correspondence: Dr. Elisa Maria Jukemura. Rua Napoleão de Barros, 690, 2o. andar. São Paulo-SP Brazil. Zip code: 04024-002. Phone: +55 11 55718935. E-mail:elisricardo@hotmail.com.

The Brazilian Journal of Infectious Diseases 2007;11(4):418-422. (C) 2007 by The Brazilian Journal of Infectious Diseases and Contexto Publishing. All rights reserved.
Acinetobacter baumannii [7], seen in 1998; more recently, Pseudomonas aeruginosa became one of the most common pathogens, mainly in the ICU setting. These latter organisms posed a substantial therapeutic dilemma, as they also became resistant to carbapenems, therefore making polymixin the only therapeutic choice for them.

In an attempt to reverse the trend of increasing antibiotic resistance, we conducted a prospective interventional study in patients who developed any infection defined as an ICU infection.

The main goal of our study was to determine whether or not substitution of third-generation cephalosporins by piperacillin/tazobactam can decrease the prevalence of Klebsiella spp. and Enterobacter spp. resistance to cephalosporins, so that carbapenems would be less frequently prescribed, in order to diminish their selective pressure on $P$. aeruginosa and Acinetobacter spp.

\section{Materials and Methods}

Hospital São Paulo is a tertiary university hospital in São Paulo, Brazil, with 624 beds, of which 40 are used for the adult Intensive-Care Unit (ICU). Our study was conducted in a 16bed general ICU that provides treatment for acutely-ill patients. All patients admitted to that ICU from September 1999 to December 2000 and who were under mechanical ventilation for more than 48 hours were included in the study. Patients who did not stay in the ICU for 48 hours were excluded from the study. All patients who were in the ICU during the study period received the proposed antibiotic for every infection believed to be due to Gram-negative bacilli in any site. 
The following data were recorded at admission: age, sex, pre-existing diseases and APACHE II score. Subsequently, the numbers of days in the ICU and mortality rates were monitored.

Due to the need for intervention, the study had a "beforeafter" design, as follows:

1. First period (from September, 1999, to February, 2000): ceftazidime was used as the antibiotic of choice for empirical treatment of suspected Gram-negative bacilli infections.

2. Second period (from August 2000, to December 2000): piperacillin/tazobactam was used for the same indication, replacing ceftazidime.

One of the investigators made daily rounds with the ICU team to ensure understanding and compliance to the study protocol. Antibiotic consumption, nosocomial infection, and microbiologic colonization were analyzed in both periods, as described below.

Some antibiotics were selected for analysis: ceftriaxone, ceftazidime, cefepime, imipenem, amikacin, ciprofloxacin and piperacillin/tazobactam. Consumption of these antibiotics was measured by using the defined daily dose (DDD), as proposed by the WHO [8]. The DDD per 1000 patient-days $\left(\mathrm{DDD}_{\mathrm{N}}\right)$ was obtained according to:

$$
\begin{aligned}
& D D D=\frac{\text { Antibiotic consumption in grams }}{\text { "Defined Daily Dose" }} \\
& \text { and } \\
& D D D_{N}=\frac{\text { DDD }}{\text { No.Patients-Day }} \times 1000
\end{aligned}
$$

Data on nosocomial infection and on ventilatorassociated pneumonia (VAP) were obtained according to NNIS methodology, defined by the Centers for Disease Control and Prevention [9]. A diagnosis of ventilatorassociated pneumonia was considered by the infectious diseases team whenever a new or progressive roentgenographic infiltrate developed in conjunction with two or more of the following clinical criteria: fever, leukocytosis and purulent tracheal aspirate.

One of us (EMJ) was responsible for collecting lower tracheal aspirates, under sterile conditions, of all patients on the second day of mechanical ventilation; this was repeated every week that patients were maintained on mechanical ventilation. The material was stored for posterior culture using classic methods of identification; susceptibility tests were performed by using National Committee for Clinical Laboratory Standards (NCCLS) disc diffusion standards [10]. Discs with ceftazidime and amoxicillin/clavulanate were used to check for ESBL in suspected isolates.

Computer-based antibiogram records were used to determine the prevalence of resistance to amikacin, ceftriaxone, ceftazidime, cefepime, ciprofloxacin, imipenem and piperacillin/ tazobactam.
Statistical Analysis

The student's t test was used to compare antibiotic consumption and hospital-infection rates between the two periods. Survival analysis of patient outcomes (discharge from ICU or death) was performed by using a Cox-rank test for the two periods. A Chi-square test was used to compare periods in terms of prevalence of pathogens and susceptibility profile of the isolated microorganisms. The statistical significance level adopted was 5\%. Analyses were performed using STATISTICA for Windows $4.5^{\mathrm{TM}}$. The study was submitted to the Ethics Committee of São Paulo Hospital and it was approved.

\section{Results \\ Patient Characteristics}

Eighty-seven and 66 patients met the criteria for analysis in the first and second periods, respectively. There were no significant differences in the clinical and epidemiological characteristics of the patients between the periods (Table 1). The survival analyses showed no significant difference in the length of stay in ICU between the periods ( $p=0.19$, Figure 1 ). In addition, the probability of death did not differ between periods ( 58.6 and $59.0 \%$, respectively $(\mathrm{p}=0.91)$.

\section{Changes in Antibiotic Use}

There was no significant variation in the consumption of most of the antibiotics (Table 2). However, there was a significant reduction in the use of third-generation cephalosporins and a significant increase in piperacillin/ tazobactam use.

\section{Hospital Infection Surveillance}

The nosocomial infection rate decreased from an average of 31.75/1000 patient-days in the first period to $18.99 / 1,000$ patient-days in the second period. This was mainly due to the decrease in ventilator-associated pneumonia (VAP) rates, decreasing from 19.93 VAP/1000 patient-days to 5.13 VAP/ 1,000 patient-days $(\mathrm{p}<0.0001$, Figure 2$)$.

\section{Microbiological Surveillance}

Microorganisms obtained from tracheal secretions were analyzed. These organisms were collected from 153 patients who met the criteria for sampling. There were decreases in the prevalence of $K$. pneumoniae and $P$. mirabilis and an increase in the prevalence of Pseudomonas aeruginosa (Table 3).

The rate of resistance of $K$. pneumoniae to ceftriaxone and ceftazidime decreased from the first to the second period ( 0.78 versus 0.07 and 0.97 versus $0.21, p<0.0001$, respectively). When these bacteria were tested for production of ESBL enzymes, $87.9 \%$ were positive in the first period and $22.2 \%$ in the second period $(p=0.0003)$. Piperacillin/tazobactam was not available in the first period, but its use in the second period did not increase the resistance of $K$. pneumoniae to it $(0.31 \mathrm{x}$ $0.14, \mathrm{p}=0.2$ ). 
Table 1. Baseline and clinical characteristics of the cohort of patients in the intensive care unit

\begin{tabular}{|c|c|c|c|}
\hline Characteristics & $\begin{array}{l}\text { First period } \\
\qquad(\mathbf{N}=87)\end{array}$ & $\begin{array}{l}\text { Second period } \\
\qquad(\mathrm{N}=66)\end{array}$ & p value \\
\hline Age, years* & 52.44 & 54.29 & 0.75 \\
\hline \multicolumn{4}{|l|}{ Sex } \\
\hline Male n (\%) & $47(54)$ & $40(60)$ & \\
\hline Female n $(\%)$ & $40(46)$ & $26(40)$ & 0.41 \\
\hline Length of stay in intensive care (days) & 19.46 & 26.06 & 0.08 \\
\hline \multicolumn{4}{|l|}{ Underlying diseases } \\
\hline Congestive heart failure n (\%) & $22(25.28)$ & $15(22.73)$ & 0.86 \\
\hline Chronic pulmonary disease $\mathrm{n}(\%)$ & $5(5.74)$ & $4(6.06)$ & 0.79 \\
\hline Malignancy n (\%) & $16(18.40)$ & $18(27.27)$ & 0.26 \\
\hline Stroke n $(\%)$ & $8(9.20)$ & $2(3.03)$ & 0.21 \\
\hline Traumatism n (\%) & $8(9.20)$ & 9 (13.64) & 0.54 \\
\hline Metabolic diseases n (\%) & $8(9.20)$ & $2(3.03)$ & 0.23 \\
\hline No underline diseases n (\%) & $15(17.24)$ & $13(19.70)$ & 0.85 \\
\hline Hepatic disorder & $5(5.74)$ & $3(4.54)$ & 0.73 \\
\hline \multicolumn{4}{|l|}{ Admission diagnoses } \\
\hline Post-operative neurosurgery & $22(25.28)$ & 9 (13.64) & 0.11 \\
\hline Post-operative cardiac surgery & $4(4.60)$ & $1(1.51)$ & 0.54 \\
\hline Post-operative pulmonary surgery & $5(5.74)$ & $2(3.03)$ & 0.68 \\
\hline Post-operative abdominal surgery & $20(23.00)$ & $23(34.85)$ & 0.15 \\
\hline Other surgeries & $4(4.60)$ & $7(10.61)$ & 0.26 \\
\hline Cardiac & 17 (19.54) & $5(7.57)$ & 0.06 \\
\hline Respiratory & $9(10.34)$ & $13(19.70)$ & 0.16 \\
\hline Neurological & $4(4.60)$ & $23.03)$ & 0.94 \\
\hline Other clinical disorders & $2(2.30)$ & $4(6.06)$ & 0.44 \\
\hline APACHE II Score ${ }^{\S}$ & 19.37 & 17.31 & 0.10 \\
\hline
\end{tabular}

Table 2. Antibiotic consumption, in $\mathrm{DDD}_{\mathrm{N}}$, in the first and second periods

\begin{tabular}{lccc}
\hline Antibiotic & First period & Second period & $\mathbf{p}^{*}$ \\
\hline Amikacin & 35.25 & 7.36 & 0.095 \\
Ceftriaxone & 73.50 & 15.80 & 0.001 \\
Ceftazidime & 34.83 & 0.85 & 0.004 \\
Cefepime & 11.17 & 6.60 & 0.39 \\
Ciprofloxacin & 50.95 & 24.70 & 0.561 \\
Imipenem-cilastatine & 58.91 & 64.00 & 0.832 \\
Piperacillin-tazobactam & - & 157.07 & 0.0002 \\
\hline
\end{tabular}

* Student $\mathrm{T}$ test.

Table 3. Frequency of tracheal isolate results in the first and second periods

\begin{tabular}{lccc}
\hline Microorganism & First period $(\%)$ & Second period $(\%)$ & $\mathbf{p}^{*}$ \\
\hline Sterile & $13(5)$ & $9(4)$ & 0.76 \\
S. aureus & $24(9)$ & $16(8)$ & 0.67 \\
K. pneumoniae & $43(16)$ & $14(7)$ & 0.004 \\
A. baumannii & $45(17)$ & $34(17)$ & 0.94 \\
P. aeruginosa & $89(33)$ & $94(46)$ & 0.005 \\
Enterobacter spp. & $13(5)$ & $8(4)$ & 0.7 \\
Serratia spp. & $10(4)$ & $3(1)$ & 0.13 \\
P. mirabilis & $13(5)$ & $2(1)$ & 0.036 \\
Other bacteria & $16(6)$ & $19(9)$ & 0.23 \\
Candida spp. & $3(1)$ & $5(2)$ & 0.16 \\
Total & 269204 & & \\
\hline
\end{tabular}

$*^{*} \chi$-square test, with Yates correction when indicated. 
Figure 1. Survival analysis during intensive care unit stay of patients in the two periods.

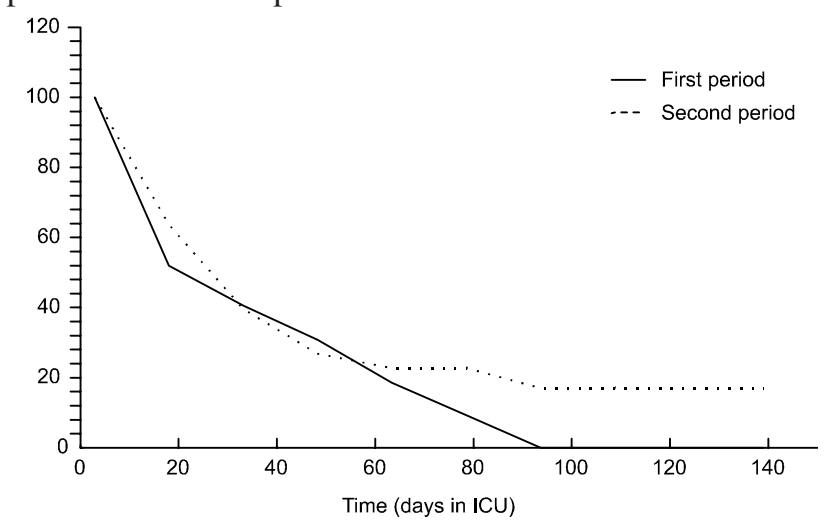

There were increases in $P$. aeruginosa resistance to piperacillin/tazobactam $(0.60$ versus $0.76, \mathrm{p}=0.02)$, meropenem $(0.33$ versus $0.45, \mathrm{p}<0.0001)$ and ciprofloxacin $(0.69$ versus $0.78, \mathrm{p}<0.0001)$ from the first to the second periods. Increased resistance was also observed to ceftazidime $(0.67$ versus $0.86, \mathrm{p}=0.003)$.

Resistance to other Gram-negative bacilli (including $A$. baumannii and Enterobacter spp.) and to other antimicrobials did not differ from the first to the second period.

\section{Discussion}

The increasing dilemma posed by the treatment of multiresistant Gram-negative bacteria emphasizes the importance of interventional measures directed towards minimizing this problem.

We tested the hypothesis that an intervention designed to reduce the incidence of ESBL and chromosome-induced beta-lactamase (class 1 or AmpC)-producing bacteria would make the use of carbapenems less necessary, and therefore would allow better control of the emergence of multi-resistant A. baumannii and $P$. aeruginosa strains, which are frequently seen in infections related to health care in ICU settings [5]. To address this issue, we proposed a prospective-interventional study to evaluate the impact of changing empirical antibiotic treatment of nosocomial VAP from third-generation cephalosporins to piperacillin/tazobactam, evaluated not only with hospital microbiology, but also by examining clinical and epidemiological aspects.

The intervention took place in the general ICU of Hospital São Paulo, because antimicrobial resistance is especially important in that unit. Recorded data on bacteremia isolates showed a high rate of $P$. aeruginosa resistance to ceftazidime (37.5\%), ciprofloxacin $(71.5 \%)$ and imipenem $(75 \%)$. Acinetobacter spp. was also resistant to ceftazidime in $93.2 \%$ of isolates [11] These findings were expected because several risk factors for antimicrobial resistance had been previously described in ICU patients $[2,12,13]$, including acutely and severely-ill patients, widespread use of empirical polyvalent antimicrobial therapy and frequent need for invasive devices and procedures.
Figure 2. Rates of ventilator-associated pneumonia (VAP) in the first and second periods, considering all cases.

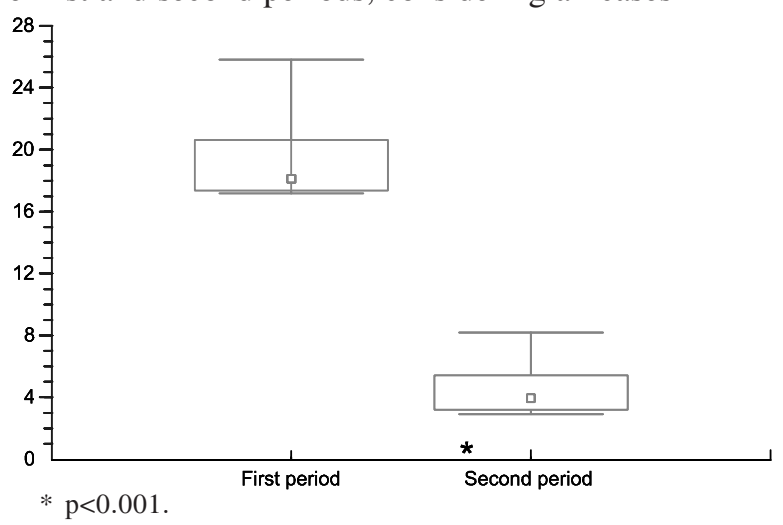

The demographic and epidemiological profiles of the patients attended in the ICU did not change during the study and the survival analysis showed no difference in the length of stay in the ICU between the periods. In addition, there was no difference in mortality from all causes. These findings allow us to conclude that bias in the intended analysis was minimized. Apparently, the study intervention caused no measurable harm to the patients attended in that ICU.

There was good adherence to the proposed intervention, as we found a significant decrease in ceftazidime and ceftriaxone usage, while piperacillin/tazobactam use rapidly increased to an average of $157.07 \mathrm{DDD}_{\mathrm{N}} /$ month.

As expected, a significant reduction in $K$. pneumoniae isolates resistant to ceftazidime was observed from one period to the other, as well as a reduction in the overall incidence of $K$. pneumoniae. These phenomena had been described earlier [9-11] in reports of outbreaks of resistant K. pneumoniae controlled by restriction of the use of third-generation cephalosporins.

Control of chromosome-induced (class 1 or AmpC) betalactamase production was also expected, since Gram-negative bacteria develop resistance to cephalosporins when exposed to third-generation cephalosporins. However, we were not able to prove this. We found a significant decrease in the incident of $P$. mirabilis, but no change in its susceptibility profile, as we also found for other Gram-negative bacteria. There is some evidence [4] that the resistance of Enterobacter spp. is reversible by restricting the use of third-generation cephalosporins. As our interventional period was only five months, it may have been too short to reverse a resistance that was not very frequent in the first period.

There was no increase in the use of carbapenems, as opposed to the findings of Rahal et al. [13], who found a significant increase in the use of this class of antibiotic after the substitution of ceftazidime in their institution. Patterson et al. [11] also evaluated the use of imipenem after the substitution of ceftazidime with piperacillin/tazobactam, demonstrating no difference after the changing of antibiotics. However, the rate of use of imipenem in our ICU was very 
high when compared to that reported from other institutions. In one report [15] from the ICARE surveillance project, for instance, the rate of imipenem use was only 10 DDD per 1,000 patient-days, six times less than what we found. This may justify the increased frequency of $P$. aeruginosa, along with the increase in its resistance to imipenem. Troillet et al. [18] analyzed the risk factors associated with the development of imipenem-resistant $P$. aeruginosa and concluded that the most important one was previous use of this antibiotic (OR 15.4; 95\% CI 4.0-58.9). This is consistent with what we found in our study, as there was an increasing incidence of imipenemresistant Pseudomonas strains associated with the extremely high rate of use of this antibiotic, which might have resulted in high selective pressure on those microorganisms. Reducing carbapenem use was not the focus of our study. Additional restriction in carbapenem use would probably be necessary in order to reduce the frequency of resistant $P$. aeruginosa.

Even with the increased incidence of resistant $P$. aeruginosa, we observed an important reduction in overall infection rates, resulting basically from a decrease in the ventilator-associated pneumonia rates. Other researchers $[16,17]$ also suggested that antibiotic changes are effective measures in VAP control.

In conclusion, although this was not a randomized study, the change in empirical antibiotic treatment from thirdgeneration cephalosporins to piperacillin/tazobactam was effective in reducing $K$. pneumoniae and $P$. mirabilis incidence and in reducing ventilator-associated pneumonia rates, since no other intervention to reduce the occurrence of this event was used during our study. In addition, it also promoted a significant reduction in the frequency of ceftazidime-resistant $K$. pneumoniae isolates.

\section{Acknowledgements}

The writers thank the Committee for Control of Infections of Hospital São Paulo for their support in this research.

\section{References}

1. Baquero F., Negri M.C., Blázquez J., et al. Antibiotic-selective environments. Clin Infect Dis 1998;27(S1):S5-11.

2. Flaherby J.P., Weinstein R.A. Nosocomial infection caused by antibiotic-resistant organisms in the intensive-care unit. Infect Control Hosp Epidemiol 1996;17:236-48.
3. Pitout J.D., Sanders C.C., Sanders W.E. Antimicrobial resistance with focus on beta-lactam resistance in Gram-negative bacilli. Am J Med 1997;103(1):51-9.

4. Smith D.S. Decreased antimicrobial resistance after changes in antibiotic use. Pharmacotherapy 1999;19(8):129S-32S.

5. Bernstein J.M., Campbell G.D. Treatment of pneumonia and its implication for antimicrobial resistance. Chest 1999;115(3):1S-2S.

6. Gales A.C., Jones R.N., Sader H.S., et al. Antimicrobial Susceptibility of Klebsiella pneumoniae Producing Extended-Spectrum betalactamase (ESBL) Isolated in Hospitals in Brazil. Braz J Infect Dis 1997;(4):196-203.

7. Martins S.T., Wey S.B., Medeiros E.A., et al. Application of control measures for infections caused by multi-resistant Gram-negative bacteria in intensive care unit patients. Mem Inst Oswaldo Cruz 2004;99(3):331-4.

8. WHO Collaborating Center for Drug statistics methodology. Anatomical Therapeutic Chemical (ATC), Classification index with Defined Daily Doses (DDD), Oslo, Norway; 2000.

9. Emori T.G., Gaynes R.P., Hughes J.M., et al. National Nosocomial Infections Surveillance System (NNIS): description of surveillance methods. Am J Infect Control 1991;19:19-36.

10. NATIONAL COMMITTEE FOR CLINICAL LABORATORY STANDARDS (NCCLS) Performance standards for antimicrobial suceptibility testing. Wayne, PA, 2001; eleventh informational supplement M100-S11.

11. Jukemura E.M., Ujivari S., Pereira C.A., et al. Secular tendencies in bloodstream infections. Infect Control Hosp Epidemiol 2000;21(2):95.

12. Meyer K.S., Urban C., Rahal J.J., et al. Nosocomial outbreak of Klebsiella infection resistant to late-generation cephalosporins. Ann Intern Med 1993;119:353-8.

13. Rahal J.J., Urban C., Lim M., et al. Class restriction of cephalosporin use to control total cephalosporin resistance in nosocomial Klebsiella. JAMA 1998;280(14):1233-7.

14. Patterson J.E., Hardin T.C., Jorgensen J.H., et al. Association of antibiotic utilization measures ad control of multiple-drug resistance in Klebsiella pneumoniae. Infect Control Hosp Epidemiol 2000;21(7):455-8.

15. Frikin S.K., Gaynes R.P., Tenover F.C., et al. Surveillance of antimicrobial use and antimicrobial resistance in United States hospitals: project ICARE phase 2. Clin Infec Dis 1999;29:245-52.

16. Kollef M.H., Murphy D., Fraser V., et al. Schedule change of antibiotic classes. A strategy to decrease the incidence of ventilator-associated pneumonia. Am J Respir Crit Care Med 1997; 156 : $1040-8$.

17. Gruson D., Gbikpi-Benissan G., Cardinaud J.P., et al. Rotation and restricted use of antibiotics in a medical intensive care unit. Impact on the incidence of ventilator-associated pneumonia caused by antibiotic-resistant Gram-negative bacteria. Am J Respir Crit Care Med 2000;162(3Pt1):837-43.

18. Troillet N., Siamore M.H., Carmeli Y. Imipenem-resistant Pseudomonas aeruginosa: risk factors and antibiotic susceptibility patterns. Clin Infec Dis 1997;25(5):1094-8. 